\title{
A Simple Approach to Design Recursive Digital Differentiators and Integrators
}

\author{
D. K. Upadhyay \\ Div. of ECE, NSIT Dwarka \\ New Delhi, India
}

\author{
R. K. Singh \\ Uttarakhand Technical University \\ Dehradun, India
}

\begin{abstract}
In this paper, a simple approach is proposed to design recursive digital differentiators and integrators by applying the concept of time-constant analysis. The time-constant combined with the magnitude response describes the system behavior of a digital differentiator and an integrator. Further, new recursive digital differentiator and integrator designs of first-order systems are also obtained for more accurate or comparable magnitude responses as compared to the existing designs of higher-order systems over wideband. These designs are more suitable for control systems and signal processing applications.
\end{abstract}

\section{Keywords}

Digital differentiator, Digital integrator, Percentage relative error, Recursive, Time-constant, Wideband

\section{INTRODUCTION}

Differentiators and integrators are useful systems to determine and estimate the time derivatives and integrals of a signal. These systems have several applications in signal processing, control systems, Radar engineering, bio-medical engineering and in a host of other practical systems. The frequency response of an ideal differentiator and an integrator are given by

$$
\begin{gathered}
H_{d}(j \omega)=K_{d}(j \omega) \\
H_{i}(j \omega)=\frac{K_{i}}{j \omega}
\end{gathered}
$$

Where $j=\sqrt{-1}, \quad K_{d}$ is the proportional constant of differentiator, $K_{i}$ is the proportional constant of integrator and $\omega$ is the angular frequency in radians. Many techniques have been developed to design digital differentiators and integrators, generally for $K_{d}=K_{i}=1$ by using recursive or non-recursive methods in the study of digital signal processing (DSP) [1-23]. In general, the recursive digital differentiators have been obtained by inverting the transfer functions of recursive digital integrators with suitable modifications. Initially, recursive digital integrators have been designed by performing a simple linear interpolation between the magnitude responses of the classical rectangular, trapezoidal and Simpson digital integrators [1-6]. Later, Al-Alaoui has proposed several designs of recursive digital differentiators and integrators by using various techniques for lower percentage relative errors as in [7-10]. A linear programming optimization approach is also proposed to design recursive digital integrators and differentiators [11]. Later, Ngo has proposed wideband digital integrator and differentiator of third-order based on Newton-Cotes integration rule. These designs approximate the ideal integrator or differentiator over the whole Nyquist band with nearly 5 percent maximum percentage relative errors (MPREs) in magnitude responses over the full Nyquist band [12]. Pei-Hsu [13] and Gupta-Varshney-Viswewaran [14] have also developed new designs of recursive digital differentiators. Then, Gupta-Jain-Kumar have proposed another wideband digital integrator and differentiator for lower relative errors [15]. Further, Al-Alaoui has also proposed 2-segment, optimized 3segment and optimized 4-segment digital integrators and differentiators [16]. Thereafter, Gupta-Jain-Kumar have also proposed recursive wideband digital integrators and differentiators for maximum percentage relative error of $3 \%$ over wideband [17]. Generally, all the existing wideband designs have higher-order systems. Therefore, the design of recursive wideband digital differentiators and integrators by using the lower-order systems is the main issue in current research environment. Recently, Upadhyay has also proposed recursive wideband digital differentiators of $2 \%$ maximum percentage relative errors almost over the full Nyquist band [18]. Non-recursive finite impulse response (FIR) digital differentiators have been designed for highly accurate magnitude response using various techniques [19-23]. However, non-recursive wideband digital differentiators comparatively have higher-order but satisfying the linear phase requirement over the whole Nyquist band. An important aspect of these investigations is that the exploration focused on the digital differentiators and integrators for $K_{d}=K_{i}=1$ over wide bands. To overcome these limitations, there is the possibility to design digital differentiators and integrators for any value of $K_{d}$ or $K_{i}$. Such type of designs may be more powerful to design more accurate digital Proportional-Derivative (PD), Proportional-Integral (PI) and Proportional-Integral-Derivative (PID) controllers in control systems. Sometimes, it is also possible that the few designs of non-unity proportional constant may be more accurate compared to the existing designs of unity proportional constant. Therefore, the more accurate design of recursive digital differentiator and integrator for unity proportional constants may be derived by using the non-unity proportional constant design with a constant multiplier. The time-constant control analysis of microwave differentiators is described by Tsai-Wu [24]. 
In this paper, the time-constant approach is discussed to characterize the performance of digital differentiators and integrators and it also serves as an important factor to derive more accurate recursive digital differentiators and integrators for unity or non-unity time-constants over wideband. This approach is applied over the existing digital differentiator of first-order system. All the figures are obtained by using MATLAB.

This paper is organized as follows: the time-constant analysis of digital differentiators and integrators is discussed in Section 2 and the design methodology of recursive wideband digital differentiators and integrators for required time-constants is discussed in Section 3. Further, the comparisons of proposed differentiator design with the existing designs of higher-order are given in Section 4. The conclusions are given in Section 5.

\section{TIME-CONSTANT ANALYSIS}

The time-constant analysis determines the transient behavior of a differentiator or integrator in the time-domain. As a consequence, it affects the frequency response of the system. The frequency responses of the differentiator and integrator, formed by an operational amplifier and a resistor-capacitor ( $R-C$ ) circuit are given by (3) and (4) respectively. Hence, the time-constants $(\tau)$ of differentiators and integrators are defined by (5) and (6) respectively [24]. Similarly, the timeconstant of digital differentiator and integrator can be expressed by (7) and (8) respectively. It can be easily seen that the timeconstant and proportional constant are exactly same parameters for differentiators but are inverse in case of integrators.

$$
\begin{aligned}
& H_{d}(j \omega)=-j \omega R C \\
& H_{i}(j \omega)=\frac{1}{j \omega R C} \\
& \tau_{d}=K_{d}=\frac{\left|H_{d}(j \omega)\right|}{\omega} \\
& \tau_{i}=\frac{1}{K_{i}}=\frac{1}{\omega\left|H_{i}(j \omega)\right|} \\
& \tau_{d}=K_{d}=\frac{\left|H_{d}\left(e^{j \Omega}\right)\right|}{\Omega} \\
& \tau_{i}=\frac{1}{K_{i}}=\frac{1}{\Omega\left|H_{i}\left(e^{j \Omega}\right)\right|}
\end{aligned}
$$

Where $\tau_{d}$ and $\tau_{i}$ are the time-constants of differentiator and integrator; $H_{d}\left(e^{j \Omega}\right)$ and $H_{i}\left(e^{j \Omega}\right)$ are the frequency responses of digital differentiator and integrator.

\section{DESIGN METHODOLOGY}

In $[19,24]$, the continuous-time variable $S$ in terms of the discrete-time variable $z$ is given by

$$
s=H(z)=\frac{2}{T} \frac{\left(1-z^{-1}\right)}{\left(1+\alpha z^{-1}\right)}
$$

Where $T$ is the sampling period, $\alpha$ is a real constant and $z^{-1}$ represents a unit time delay. It is obtained that the eq. (9) behaves as a wideband digital differentiator for the values of $2 / T=1.1517$ and $\alpha=0.2668$, after the minimization of absolute percentage relative error as defined in (10) by using the linear programming approach [11]. This optimization also confirms that all the obtained designs must have the pole location inside the unit circle. This design of wideband digital differentiator ( $K_{d}=1$ or unity time-constant) is given in (11). Similarly, we have also obtained the designs of recursive wideband digital differentiators for different values of proportion constants $\left(K_{d}\right)$ or time-constants $\left(\tau_{d}\right)$ as 1.1, $1.1595,1.2$ and 1.3; the corresponding digital differentiator designs are given in (12-15) respectively. Table 1 shows the values of $2 / T$ and $\alpha$ for the obtained recursive digital differentiators of required time-constants.

$$
\begin{gathered}
A P R E=100\left[\frac{|| H\left(e^{j \Omega}\right)\left|-K_{d} \Omega\right|}{K_{d} \Omega}\right] \\
H_{1}(z)=\frac{1-z^{-1}}{0.8683+0.2317 z^{-1}} \\
H_{1.1}(z)=\frac{1-z^{-1}}{0.79+0.1534 z^{-1}}
\end{gathered}
$$

$$
\begin{gathered}
H_{1.16}(z)=\frac{1-z^{-1}}{0.7566+0.1203 z^{-1}} \\
H_{1.2}(z)=\frac{1-z^{-1}}{0.7353+0.0987 z^{-1}}
\end{gathered}
$$

$$
H_{1.3}(z)=\frac{1-z^{-1}}{0.7022+0.0656 z^{-1}}
$$

$$
H_{\text {imp }}(z)=\frac{1-z^{-1}}{0.6525+0.1038 z^{-1}}
$$


Table 1. Time-constants of recursive digital differentiators

\begin{tabular}{|c|c|c|}
\hline $\begin{array}{c}\text { Time-constant } \\
\left(\tau_{d}\right) \text { or } K_{d}\end{array}$ & $2 / T$ & $\alpha$ \\
\hline 1.0 & 1.1517 & 0.2668 \\
\hline 1.1 & 1.2658 & 0.1942 \\
\hline 1.1595 & 1.3217 & 0.159 \\
\hline 1.2 & 1.36 & 0.1342 \\
\hline 1.3 & 1.4241 & 0.0934 \\
\hline
\end{tabular}

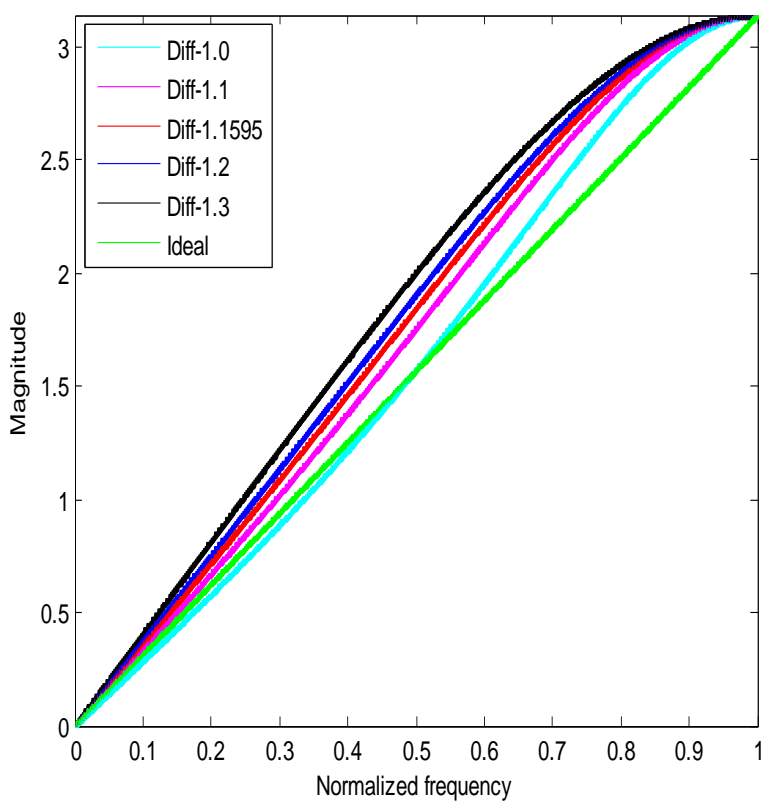

Fig 1: Magnitude responses of obtained recursive digital differentiators for required time-constants $\left(\tau_{d}\right)$

The magnitude responses of the obtained recursive digital differentiators for required time-constants are shown in Figure 1. Further, the percentage relative errors (PREs) of the obtained recursive digital differentiators from the ideal differentiators of corresponding time-constants are shown in Figure 2.

From Figures 1 and 2, it is observed that the few optimized designs for non unity time-constants may be wideband with lower percentage relative error compared to the unity timeconstant design as in (11). As an example, the proposed design of proportion constant $K_{d}=1.1, H_{1.1}(z)$ has not more than $3.6 \%$ relative error almost over the $95 \%$ of Nyquist bandwidth and the another proposed design of proportion constant $K_{d}=1.2, H_{1.2}(z)$ has not more than $1.3 \%$ relative error almost over the $70 \%$ of Nyquist bandwidth, while unity timeconstant design has nearly $9 \%$ maximum percentage relative error over the full Nyquist band. It is also observed that one another design of $K_{d}=1.1595, H_{1.16}(z)$ has not more than $1.64 \%$ relative error almost over the $80 \%$ of Nyquist bandwidth. Hence, the obtained recursive digital differentiator designs $H_{1.1}(z), H_{1.16}(z)$ and $H_{1.2}(z)$ as defined in (1214) are more preferable to design PD controllers. It is also noticed that the design of digital differentiator $H_{1.16}(z)$ as in (13) can also be converted to design a recursive digital differentiator of unity time-constant. The corresponding digital differentiator of unity time-constant is given in (16).

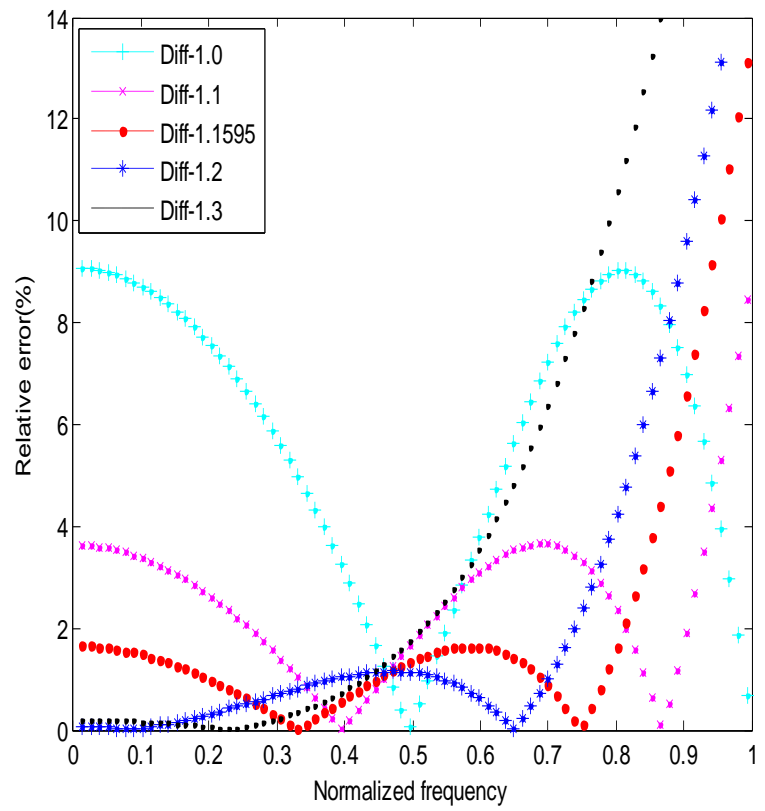

Fig 2: Percentage relative errors of the obtained recursive digital differentiators with the ideal differentiators of required time-constants

The recursive wideband digital integrators can be easily obtained by inverting the transfer functions of proposed recursive digital differentiators as in (11-16). Table 2 shows the values of proportion constants $K_{i}$ with the corresponding timeconstants for different designs of recursive digital integrators. The magnitude responses of such types of recursive wideband digital integrators for required time-constants are shown in Figure 3. Further, the percentage relative errors (PREs) of the recursive digital integrators, which are the exactly inverse of proposed recursive digital differentiators, are shown in Figure 4.

Table 2. Time-constants of recursive digital integrators

\begin{tabular}{|c|c|c|c|}
\hline $\begin{array}{c}\text { Time-constant } \\
\left(\tau_{i}\right)\end{array}$ & $T / 2$ & $\alpha$ & $K_{i}$ \\
\hline 1.0 & 0.8683 & 0.2668 & 1.0 \\
\hline 1.1 & 0.79 & 0.1942 & 0.9091 \\
\hline 1.16 & 0.7566 & 0.159 & 0.8621 \\
\hline 1.2 & 0.7353 & 0.1342 & 0.8333 \\
\hline 1.3 & 0.7022 & 0.0934 & 0.7692 \\
\hline
\end{tabular}




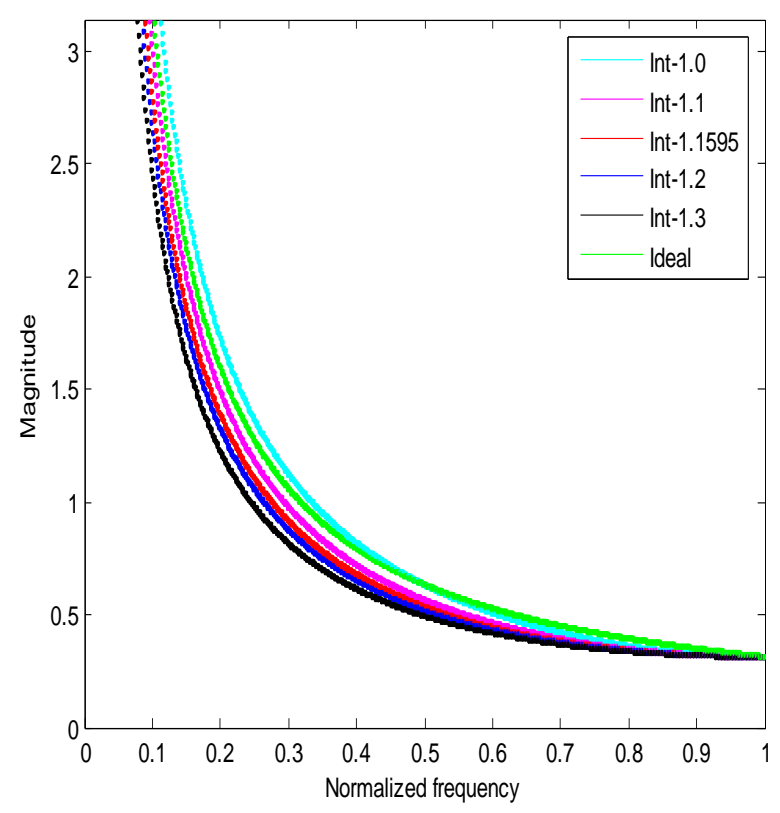

Fig 3: Magnitude responses of the recursive digital integrators for different time-constants $\left(\tau_{i}\right)$

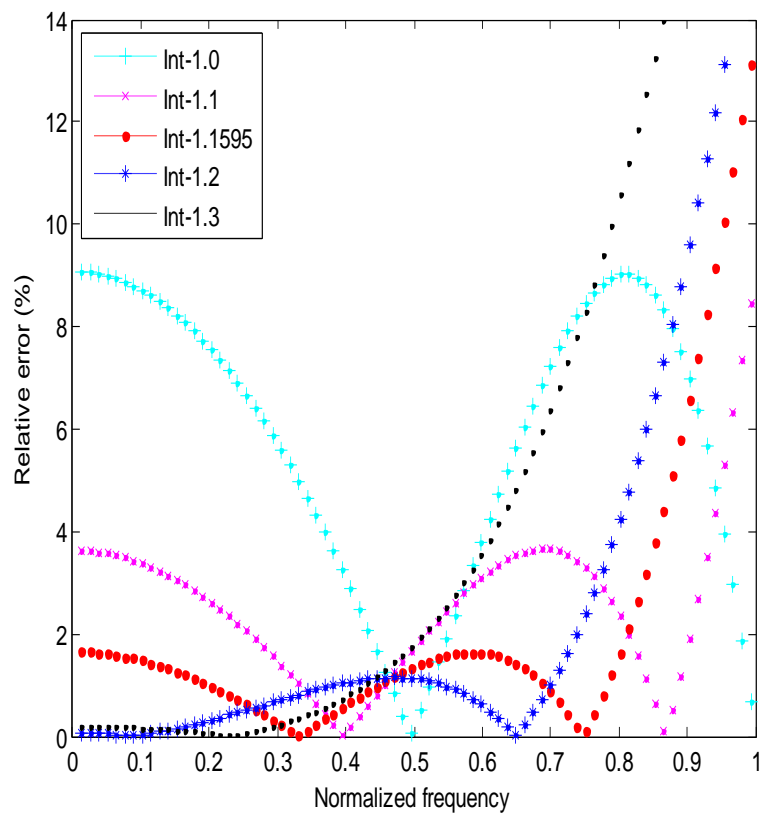

Fig 4: Percentage relative errors of the recursive digital integrators with the ideal integrators of required timeconstants

The results from Figures 3 and 4 are similar to the results of obtained recursive digital differentiators. The main difference is that the time-constant of recursive digital integrator is the inverse of the proportion constant while this is same as in the case of proposed recursive digital differentiator. These non unity time-constant designs are much preferable to design PI controllers as compared to the unity time-constant design. Further, the design of recursive digital integrator for unity timeconstant, which has not more than $1.64 \%$ relative error almost over the $80 \%$ of Nyquist bandwidth, can also be obtained by inverting the transfer function of corresponding digital differentiator as given in (16).

\section{COMPARISION}

To verify the accuracy of proposed recursive digital differentiator $H_{i m p}(z)$ as defined in (16), recently proposed Alaoui design of order two [16] and the Gupta-Jain-Kumar (GJK) design of order three [17] have been considered, the transfer functions of these designs are given in $(17,18)$ respectively. The PREs of the proposed, Al-Alaoui and the Gupta-Jain-Kumar recursive digital differentiators with the ideal differentiator of unity time-constant are shown in Figure 5.

$H_{m d}(z)=\frac{1}{0.329 T(2.663)}\left(\frac{1-z^{-1}}{\left(1+0.3755 z^{-1}\right)\left(1-0.2079 z^{-1}+0.03864 z^{-2}\right)}\right)$

$$
H_{A l-2 d}(z)=\frac{8.8438}{T}\left(\frac{1-z^{-2}}{\left(7+8.2543 z^{-1}+2.4333 z^{-2}\right)}\right)
$$

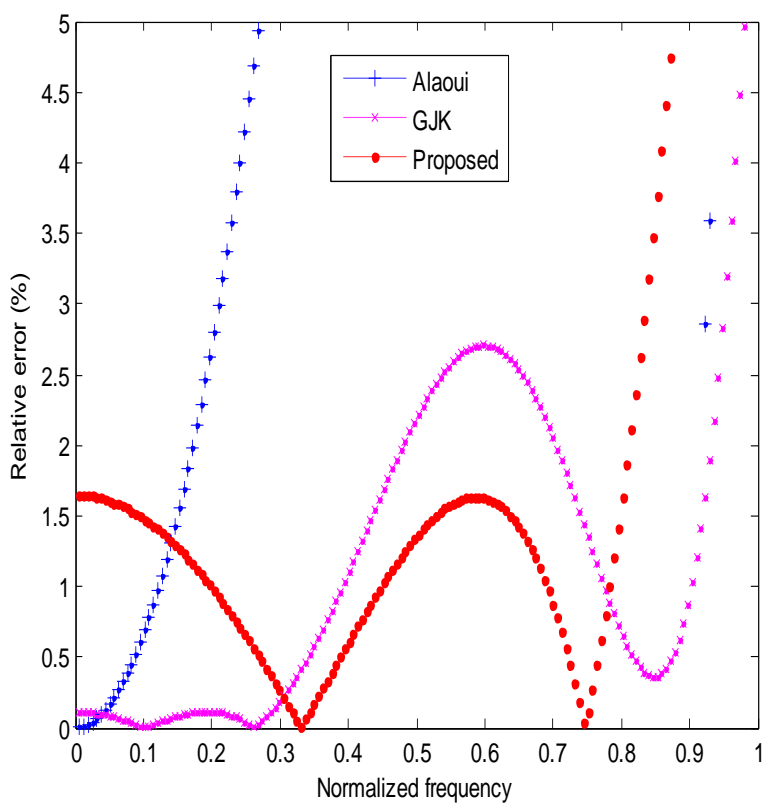

Fig 5: Percentage relative errors of proposed, Al-Alaoui and Gupta-Jain-Kumar recursive digital differentiators with the ideal differentiator of unity time-constant 
From Figure 5, it is observed that the proposed differentiator design $H_{i m p}(z)$ has not more than $1.64 \%$ relative error almost over the $80 \%$ of Nyquist band, while the Al-Alaoui design of second-order system has high amount of error at mid band and the GJK design of third-order system has 3\% relative error over wideband. Proposed integrator design is just the inverse of proposed differentiator design. Therefore, there is no need to compare the integrator design with the existing Al-Alaoui and Gupta-Jain-Kumar integrator designs.

\section{CONCLUSION}

The time-constant analysis of a digital differentiator and integrator is discussed. Then, the recursive wideband digital differentiators and integrators for different time-constants are obtained by using the proposed time-constant analysis. These designs are much useful to design more accurate digital PD and PI controllers. Further, a new recursive digital differentiator of first-order system for unity time-constant is also proposed which has maximum percentage relative error of $1.64 \%$ in magnitude response over wideband.

\section{ACKNOWLEDGMENTS}

The authors are most grateful to Prof. Raj Senani (Director, Netaji Subhas Institute of Technology, Dwarka, Delhi, India) for providing the research environment in the institute.

\section{REFERENCES}

[1] Al-Alaoui, M. A. 1988. A stable differentiator with a controllable signal to Noise ratio. IEEE Trans. IM-37, pp. 383-388.

[2] Al-Alaoui, M. A. 1992. Novel approach to designing digital differentiators. Electron. Lett., vol. 28(15), pp. 1376-1378.

[3] Al-Alaoui, M. A. 1993. Novel digital integrator and differentiator. Electron. Lett., vol. 29(4), pp. 376-378.

[4] Bihan, J. L. 1993. Novel class of digital integrators and differentiators. Electron. Lett., vol. 29(11), pp. 971-973.

[5] Al-Alaoui, M. A. 1994. Novel IIR differentiator from the Simpson integration rule. IEEE Trans. Circuits Syst. I, Fundam. Theory Appl., vol. 41(2), pp. 186-187.

[6] Al-Alaoui, M. A. 1995. A class of second-order integrators and low-pass differentiators. IEEE Trans. Circuits Syst. I, Fundam. Theory Appl., vol. 42(4), pp. 220-223.

[7] Al-Alaoui, M. A. 1997. Filling the gap between the bilinear and the backward difference transforms: an interactive design approach. International Journal of Electrical Engineering, vol. 34(4), pp. 331-337.

[8] Al-Alaoui, M. A. 2001. Novel stable higher order $s$ to $\mathrm{z}$ transforms," IEEE Trans. Circuits Syst. I, Fundam. Theory and Appl., vol. 48(11), pp. 1326-1329.

[9] Al-Alaoui, M. A. 2007. Linear phase low pass IIR digital differentiators. IEEE Trans. Signal Processing, vol. 55(2), pp. 697-706, February.

[10] Al-Alaoui, M. A. 2008. Al-Aloui operator and the new transformation polynomials for discretization of analogue systems. Electrical Engineering, vol. 90(4), pp. 455-567.

[11] Papamarkos N. and Chamzas, C. 1996. A new approach for the design of digital integrators. IEEE Trans. Circuits Syst. I, Fundam. Theory Appl., vol. 43(9), pp. 785-791.
[12] Ngo, N. Q. 2006. A New Approach for the Design of Wideband Digital Integrator and Differentiator. IEEE Transactions on circuits and systems-II, vol. 53(9), pp. 936-940.

[13] Pei, S.C. and Hsu, H.J. 2008. Fractional bilinear transform for analog to digital conversion. IEEE Trans. Signal Processing, vol. 56(5), pp. 2122-2127.

[14] Gupta, M., Varshney, P., Viswewaran G.S. and Kumar, B. 2008. Novel digital differentiator and corresponding fractional order differentiator models. Sigmap, pp. 47-54.

[15] Gupta, M., Jain M. and Kumar, B. 2009. "Wideband digital integrator," Proceedings of IEEE, IMPACT. pp. 106-108.

[16] Al-Alaoui, M. A. 2009. Novel class of digital integrators and differentiators. IEEE Trans. Signal Processing, DOI: 10.1109/TSP.2008.929668.

[17] Gupta, M., Jain, M. and Kumar, B. 2010. Recursive wideband digital integrator and differentiator. Wiley Int. J. Circ. Theor.Appl., DOI:10.1002/cta.658.

[18] Upadhyay, D. 2010. Recursive wideband digital differentiators. Electron. Lett., 46(25), pp. 1661-1662.

[19] Oppenheim, A. V., Schaffer, R. W. and Buck, J. R. 1999. Discrete time signal processing, PHI.

[20] Kumar, B. and Dutta Roy, S.C. 1988. Design of digital differentiators. Proceedings of IEEE, vol. 76(3), pp. 287 289 .

[21] Kumar, B. and Dutta Roy, S.C. 1989. Maximally linear FIR digital differentiators for high frequencies. IEEE Trans. Circuits and Systems, vol. 36(4), pp. 890-893.

[22] Sunder, S. and Ramachandran, V.1994. Design of equripple non recursive digital differentiators and Hilbert transformers using a weighted least square technique. IEEE Trans. Signal Processing, vol. 42(9), pp. 2504-2509.

[23] Mollova, G. 1999. Compact formulas for least-squares design of digital differentiators. Electron. Lett., 35(20), pp. 1695-1697.

[24] Tsai, L.-C. and Wu, Y.-T. 2009. Time-Constant Control analysis of microwave differentiators. IET Microw. Antennas Propag., vol.3, pp.1044-1050.

\section{AUTHORS PROFILE}

Dharmendra K. Upadhyay received his B.E. degree in Electronics \& Communication Engineering from Kumaon University, Nainital, India. Then, he received M.Tech. degree in Communication \& Information Systems from Aligarh Muslim University, Aligarh, India. $\mathrm{He}$ is pursuing $\mathrm{Ph} . \mathrm{D}$. degree in Electronics \& Communication Engineering Division from Uttarakhand Technical University, Dehradun, India. His field of research includes Digital Signal Processing and Microwave Engineering. He is working as Assistant Professor in Electronics \& Communication Engineering Division of Netaji Subhas Institute of Technology Delhi, India

Rakesh K. Singh received his M.Tech. degree in Electronics \& Control Engineering from BITS, Pilani (Rajasthan), India. Then, he received Ph.D. degree in Electronics Engineering from Allahabad University, Allahabad, India. His field of research includes Digital Signal Processing, VLSI and Image Processing. $\mathrm{He}$ is working as Professor in Electronics \& Communication Engineering Division of Uttarakhand Technical University Dehradun, India. 